\title{
A Path Analysis of Psychotic Symptoms among Persons with Schizophrenia using Methamphetamines
}

\author{
Ek-uma IMKOME ${ }^{1, *}$, Jintana YUNIBHAND ${ }^{2}$ and Waraporn CHAIYAWAT ${ }^{2}$ \\ ${ }^{I}$ Faculty of Nursing, Thammasat University, Bangkok 10330, Thailand \\ ${ }^{2}$ Faculty of Nursing, Chulalongkorn University, Bangkok 10330, Thailand
}

("Corresponding author's e-mail: ek-uma@nurse.tu.ac.th)

Received: 1 June 2017, Revised: 2 February 2018, Accepted: 3 March 2108

\begin{abstract}
Knowledge of psychotic symptoms among persons with schizophrenia influenced by methamphetamine use guides the design of nursing innovations to maximize positive patient outcomes. This cross-sectional, descriptive correlation study aimed to explore the relationships among coping, medication use self-efficacy, expressed emotions, stressful life events, social support, and social dysfunction, and to test a model that explained the influences of these factors on psychotic symptoms among persons with schizophrenia using methamphetamines.

The stress-vulnerability model for schizophrenia guided this study. A sample of 313 persons with schizophrenia using methamphetamines in psychiatric hospitals and institutes for drug abuse treatment in Thailand was recruited by multi-stage sampling and responded to a Demographic Questionnaire, Brief Psychiatric Rating Scale, Brief COPE, Self-efficacy for Appropriate Medication Use Scale, Expressed Emotional Scale, Stressful Life Events Questionnaire, and Social Dysfunction Scale. A linear structural relationship was used to test the hypothesized path model.

The hypothesized model was found to fit the empirical data and explained $54 \%$ of variance in psychotic symptoms $\left(\chi^{2}=8.28, \mathrm{df}=8, \chi^{2} / \mathrm{df}=1.0, \mathrm{GFI}=0.99\right.$, AGFI $\left.=0.96, \mathrm{RMSEA}=0.01\right)$. The highest total effect and factors directly affecting psychotic symptoms were emotionally focused coping strategies, medication use self-efficacy, social dysfunction, positively expressed emotions, and stressful life events.

The findings recommend that emotionally focused coping strategies, self-efficacy in medication use, social dysfunction, positively expressed emotions, and stressful life events were important factors that influenced psychotic symptoms in patients. Nursing interventions designed to manage these factors are crucial for reducing psychotic symptoms.
\end{abstract}

Keywords: Coping strategies, methamphetamines, path analysis, psychotic symptoms, schizophrenia, self-efficacy

\section{Introduction}

Methamphetamine abuse is common in patients with schizophrenia and dramatically deteriorates their clinical symptoms. Up to $80 \%$ [1] of schizophrenia patients use methamphetamines. Chronic methamphetamine use may result in significant anxiety, confusion, insomnia, mood disturbances, and violent behavior, with such psychotic features as paranoia, visual and auditory hallucinations, and delusions. Psychotic symptoms can sometimes last for months or years after a person has stopped methamphetamine use. Stressful life events, level of substance use, non-compliance with treatment, poor global functioning, and violence may precipitate spontaneous recurrence of methamphetamine psychosis [2]. 
http://wjst.wu.ac.th

Psychotic symptoms devastate the lives of affected persons and disrupt families. Affected individuals may withdraw from society and display regressive behavior and become unable to maintain personal hygiene, to engage with others, or to notice physical illness and pain. In the longer term, severe psychotic symptoms lead to low social functional skill and low quality of life, and stigmatize the affected individual and their loved ones [3].

The relationship between psychotic symptoms and related factors is complex. Nurses may play a significant role in treating some psychotic symptoms of schizophrenia patients, helping them to adjust to hallucinations, delusions, and associated treatments [4,5]. Nurses may also assist patients in managing side effects of psychotherapeutic drugs and in living with their symptoms functionally. Thailand is enduring increasing schizophrenia and methamphetamine use. Therapeutic efforts and nursing interventions focusing more on human response to improving patient function and well-being, and the need to better understand multiple factors that work to affect psychotic symptoms, will facilitate the design of optimally effective nursing interventions. They will also provide individualized interventions to maximize positive patient outcomes. Models testing relationships among psychotic symptoms in schizophrenia patients using methamphetamines have never before been studied in Thailand. Previous studies investigating these models in Western countries cannot be applied to Thailand because of different cultural beliefs about mental health symptoms.

To explore the correlation between psychotic symptoms and predicted variables, a model was developed and tested to explain influences on psychotic symptoms of persons with schizophrenia using methamphetamines. The results should facilitate the design of optimal nursing interventions to reduce psychotic symptoms among these patients.

\section{Conceptual framework and literature review}

The current study was guided by the Vulnerability-Stress Model of Schizophrenia [6] and focused on 2 human responses:

1) Reactions to actual health problems or illness (health-restoring responses);

2) Concerns about potential health problems (health-supporting responses).

This model determines the factors that affect schizophrenic psychotic symptoms and integrates a holistic perspective in which both biological and psychological variables explain the onset, course, and psychotic symptoms of persons with schizophrenia showing interaction among 4 factors:

(a) Personal vulnerability factors, including dopaminergic dysfunction, reduced available processing resources, autonomic hyperactivity, and schizotypal personality traits [6];

The dopaminergic dysfunction will reduce the activation of processing resources and affect tonic autonomic hyper activation. The interaction of the personal vulnerability factor and personal protectors leads the vulnerable individual to develop prodromal symptoms of schizophrenia. However, the personal vulnerability factors are associated with inherited genetic factors and/or early biological factors. These factors have been thought to contribute to vulnerability to schizophrenia and congenitally compromised brain structure and function.

Personal vulnerability factors were not included in this study because the authors focused only on the human responses to the actual or potential health problems of the population.

b) Personal protective factors, including coping skills, self-efficacy, and antipsychotic drugs; for this study, the researcher used the medical usage of self-efficacy as confidence in one's ability to perform a given task, such as taking antipsychotic medications as prescribed.

The strength of self-efficacy for appropriate antipsychotic use plays an important role in taking antipsychotics and can balance neurotransmitters in the brain, especially dopamine and norepinephrine, which leads to a decrease in both positive psychotic symptoms and negative psychotic symptoms [7].

Coping [6]: coping concerns the strategies, behaviors, or cognitive efforts of schizophrenic persons misusing methamphetamines, in terms of problem focused coping strategies, to control the emotional distress caused by an event, which is termed the emotion-focused coping strategy, and dysfunctional coping strategy. Patients with schizophrenia patients are often ill-prepared to cope with stress in their life and pressure from family members because they often lack the information-processing skills to process optimum behavioral alternatives and the social skills to put these strategies into action. 
http://wjst.wu.ac.th

In the current study, the researcher focused on human response, and the self-efficacy and antipsychotic drug factors were integrated as medication use self-efficacy. Therefore, the factors of coping and medication use self-efficacy was tested.

c) Environmental protective factors, including effective family problem solving and supportive psychosocial interventions;

Effective family problem solving means the ability of family members to solve problems; not only the individual problems of persons with schizophrenia, but also the problems of all family members, which are always related to the conditions of each individual's life, in his or her household, the neighborhood or town, and the larger community. This factor was not included in this study. Additionally, after the researcher reviewed the measurement of these factors, it was found that the constructs and items for the effective family problem-solving scale presented multicollinearity between coping and expressed emotion. Thus, in this study, effective family problem solving was excluded.

Regarding the supportive psychosocial interventions, these are interventions to treat patients. However, the focus of this study was human response. Therefore, the researcher set this variable as demographic data that was a part of the medical history of the study participants.

Social support: social support is a factor that the researcher added to the environmental protective factor of this study. Stress factors can exacerbate psychotic symptoms. Therefore, support from family, friends, medical specialists, or clinical practitioners represents a key component in helping patients to raise protective factors for the reduction of symptom severity [8].

In conclusion, environmental protective factors were factors used to explore relationships in the current study.

d) Environmental potentiates and stressors, including critical or emotionally over-involved attitudes toward patients, over-stimulating social environments, and stressful life events. There is a strong relationship in the empirical study among high expressed emotions, stressful events, and positive symptoms of the disorder, such as hallucinations and delusions $[9,10]$.

Outcome variables include social function, psychotic symptoms, and occupational function. Chosen for the conceptual framework were emotionally-focused coping strategies, problem-focused coping strategies, dysfunctional coping strategies, medical use self-efficacy, negative expressed emotions, positive expressed emotions, stressful life events, social dysfunction, and social support variables influencing psychotic symptoms among persons with schizophrenia using methamphetamines.

\section{Study aims}

1. To explore relationships among emotionally-focused coping strategies, problem-focused coping strategies, dysfunctional coping strategies, self-efficacy in medication use, negatively expressed emotions, positively expressed emotions, stressful life events, social support, social function, and psychotic symptoms in schizophrenia patients using methamphetamines.

2. Developing and testing a model to explain influences on psychotic symptoms among schizophrenia patients using methamphetamines. These include emotionally-focused coping strategies, problem-focused coping strategies, dysfunctional coping strategies,, self-efficacy in medication use, negatively expressed emotions, positively expressed emotions, stressful life events, social support, and social function.

\section{Methods}

\section{Participants}

Schizophrenia patients using methamphetamines attending an inpatient unit in 8 psychiatric and substance abuse services in Thailand were recruited using a multi-stage sampling technique. 313 samples agreed to be screened to determine eligibility to participate in the study. Inclusion criteria required samples to:

1) Be aged between 19 and 60;

2) Have a principal diagnosis of schizophrenia and evidence of methamphetamine use;

3) Have a Brief Psychotic Rating Scale score of less than 36; 
http://wjst.wu.ac.th

4) Be admitted as an inpatient;

5) Be able to communicate in Thai language;

6) Be willing to participate.

\section{Sample size determination}

The hypothesized model contained 10 observed variables, and $10 \%$ of total sample size was added to account for dropouts (Figure 1). The remaining number of samples was 220. Trying to decrease data deviation further by assuming multivariate normality, communalities became small. Multiple construct models with communalities of less than 0.5 required larger sizes for convergence and model stability. Over 300 samples were recommended [11] and 313 samples were recruited.

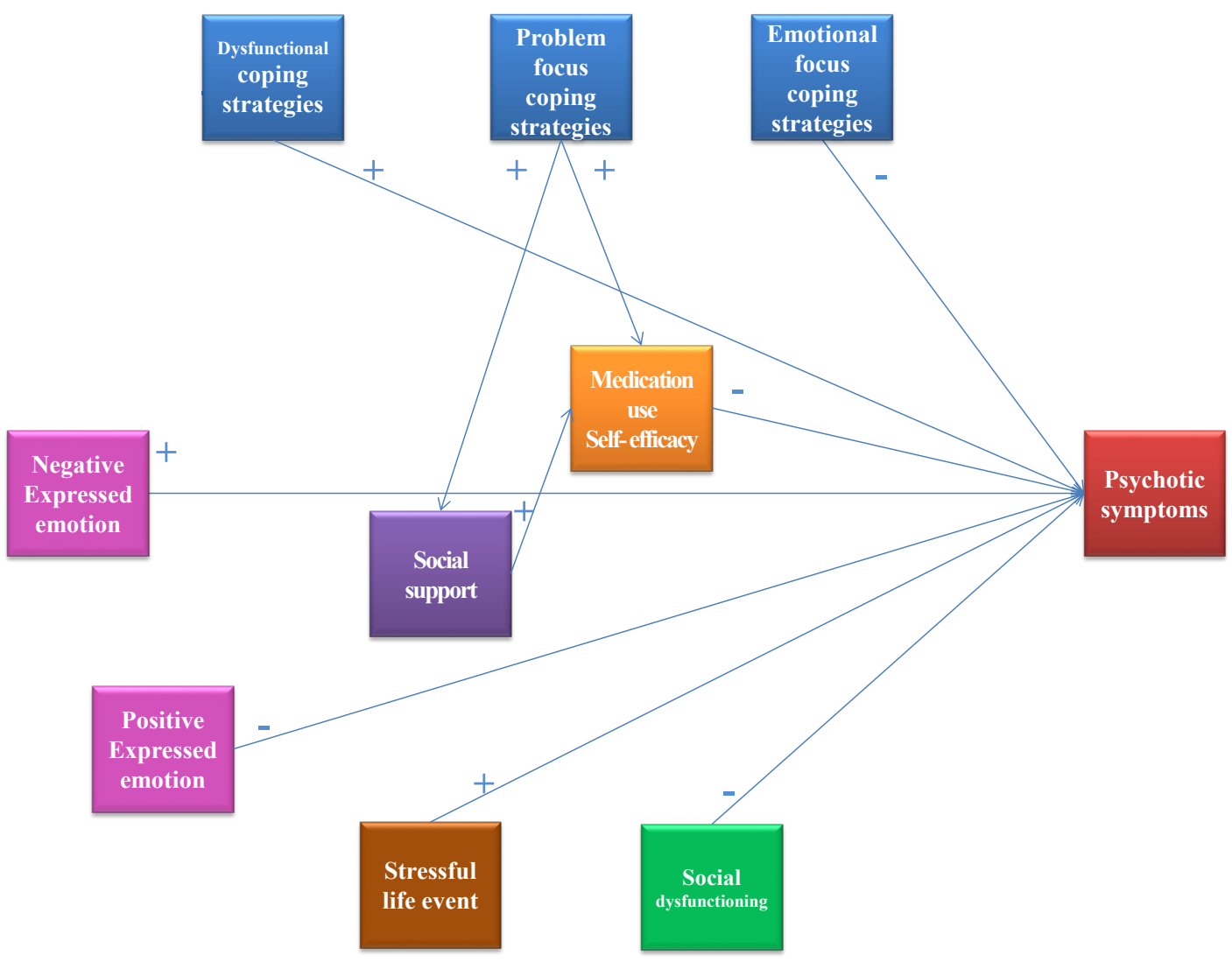

Figure 1 Hypothesized model of path analysis of psychotic symptoms in schizophrenia patients using methamphetamines, adapted from the Vulnerability-Stress Model of Schizophrenia [6].

\section{Ethical considerations}

The study was approved by the Ethics Review Committee for Research Involving Human Research Subjects, Health Science Group, Chulalongkorn University (COA. No. 053/2016). Each participant received information about the purposes, benefits, risks, and right to withdraw from the study before signing the consent form. Participants were assured of confidentiality and anonymity about participation. Confidentiality was assured by assigning a code number to each completed questionnaire rather than the subject's name, and separating returned questionnaires from signed consent forms. 


\section{Data collection}

Participant processes of informed consent included information consent and comprehension. They were asked to complete the questionnaires within from 45 to $60 \mathrm{~min}$. Questionnaires were read aloud to subjects who were not comfortable reading for themselves. When completing questionnaires, each participant was given a pill case as a gift in thanks for their participation.

\section{Instruments}

1) The Brief Psychiatric Rating Scale (BPRS) [12] was used to measure psychiatric symptoms, a semi-structured interview with an 18-item rating scale based on patient observations and verbal reports. The total scale score ranged from -18 to 126 , from "not present" to "extremely severe". In this study, the BPRS exhibited reliability $=0.98$, and intraclass correlation coefficient $=0.88$.

2) The Brief COPE [13] was translated into Thai language, a self-report with 28 items in 14 dimensions: self-distraction, active coping, denial, substance use, use of emotional support, use of instrumental support, behavioral disengagement, venting, positive reframing, planning, humor, acceptance, religion, and self-blame. Each of the 14 scales was captured by 2 items and responses on 4point scales, with options ranging from "have not been doing" to "doing this a lot." Scores ranged from 0 to 84 . The validity of the instruments in this study was assessed by 7 content experts: 2 psychiatrists, 1 psychologist, 3 nursing instructors, and 1 psychiatric nurse trained as an advanced practice nurse (APN). The Brief COPE demonstrated CVI $=1.0$, construct reliability $=0.90$, average variance extracted $=0.84$, Cronbach's alpha $=0.91$, item-total correlations ranged from 0.35 to 0.76 , and test retest $=0.96$.

3) Self-efficacy for appropriate medication use scale (SEAM) [14] with 13 items was in 2 dimensions; the first was self-efficacy for taking medications under difficult circumstances, and the second self-efficacy for continuing to take medications when circumstances of taking medication are uncertain. The Likert scale ranged from not confident to very confident. Scores ranged from 13 to 39 . The SEAM showed Cronbach's alpha $=0.91$, item-total correlations ranged from -0.07 to 0.62 , and test retest $=0.97$.

4) The Thai version of the family expressed emotional scale (TFEES) [15] was composed of 5 constructs: critical comments, hostility, positive remarks, warmth, and emotional over-involvement in interactions with family caregivers. The 16 items rated on a 4-point Likert scale ranged from "disagree" to "agree strongly." This measurement showed CVI $=0.90$, construct reliability $=0.99$, Average variance extracted $=0.90$, Cronbach's alpha $=0.88$, item-total correlations ranged from -0.33 to 0.72 , and test retest $=0.95$.

5) The stressful life events questionnaire (SLE) [16] was translated into Thai, a self-report with 2 constructs, including self-perceived frequency and intensity of stressful life events. The TSLEQ consisted of 46 items on a 6-point Likert scale, ranging from "never" to "very severe." The 11 domains covered home life, financial problems, social relations, personal conflicts, job conflicts, educational concerns, job security, loss and separation, sexual life, daily life, and health concerns. In the validity of the barriers using 7 content experts, the CVI was 1.0 , Cronbach's alpha $=0.97$, item-total correlations ranged from 0.27 to 0.92 , and test retest $=1.00$.

6) The social support questionnaire (SSQ) [17] consisted of 2 parts designed to measure informational, emotional, and tangible support. The questionnaire consisted of 7 items on 3 resources of support: 1 for information support, 4 for emotional support, and 2 for tangible support. SSQ was rated on the Likert scale ranging from "not at all" to "a great deal". Scores for 3 types of support from all sources were added to produce a total social support score. SSQ showed Cronbach's alpha $=0.93$, item-total correlations ranged from 0.38 to 0.67 , and test retest $=0.95$.

7) The social and occupational functioning scale (SOFS) [18] was translated into Thai. The SOFS is an observer rating scale comprised of 2 main components:

a) The ability to look after oneself and maintain daily activities;

b) The instrumental and social skills to manage oneself and live in the community.

Each item was rated on a 5-point Likert scale ranging from "no impairment" to "extreme impairment." This instrument showed CVI $=1.00$, Cronbach's alpha $=0.94$, item-total correlations ranging from -0.27 to 0.78 , and test retest $=0.96$. 
http://wjst.wu.ac.th

\section{Data analysis}

Descriptive statistics were applied to all variables, and data were screened for univariate normality using PRELIS in LISREL 8.72. The distributions for each variable were examined; some were skewed or displayed kurtosis. Data with non-normal distributions were transformed using a normal score. Multicollinearity and assumption of path were also tested. Linear Structural Relationship was employed for path analysis.

\section{Results}

\section{Description of the sample}

Findings revealed that the mean age of the 313 participants was 31.45 years $(\mathrm{SD}=7.83$, range $=19$ to 58$)$. They were predominantly male $(87.9 \%)$, single (66.1\%), and had completed secondary and high school education (55.0 \%). Regarding patient medical history, nearly half (47.0\%) had psychiatric illness of durations between 1 and 5 years. Over two-thirds were treated with antipsychotic drugs $(73.2 \%)$ and group therapy $(87.2 \%)$. Nearly half consumed 2 to 5 tablets of methamphetamine daily $(48.2 \%)$. The primary route of methamphetamine use was through smoking $(91.1 \%)$, and more than half of the patients $(62.3 \%)$ concurrently smoked cigarettes.

\section{Relationships between variables}

Bivariate Pearson correlations indicated that emotion-focused coping was negatively associated with psychotic symptoms $(\mathrm{r}=-0.117, \mathrm{p}<0.05)$. Problem-solving coping was not significantly associated with psychotic symptoms $(\mathrm{r}=-0.017, \mathrm{p}<0.05)$. By contrast, problem-solving coping was positively associated with social support $(\mathrm{r}=$ associated with psychotic symptoms $(\mathrm{r}=0.118, \mathrm{p}<0.05)$ and medication use self-efficacy $(r=0.210, p<0.01)$. Social support was not significantly correlated with psychotic symptoms $(r=-0.072, p<0.05)$ or stressful life events $(r=-0.091, p<0.05)$. However, social support was negatively associated with social function $(\mathrm{r}=0.098, \mathrm{p}<0.05)$, and medication use selfefficacy was negatively associated with psychotic symptoms $(r=-0.015, p<0.01)$ among schizophrenia patients using methamphetamines (Table 1).

\section{Hypothesis testing}

Factor analysis was conducted to examine factor loading for each item and goodness-of-fit indices of the measurement model and data. The results of a second-order confirmatory factor analysis (CFA) revealed that 3 measurement models had a good overall model fit (measurement model of social support: $\chi^{2}=160.13, \mathrm{df}=135, \mathrm{p}$-value $=0.07, \chi^{2} / \mathrm{df}=14.22, \mathrm{GFI}=0.95$, AGFI $=0.92$, RMSEA $=0.02$, measurement model of coping: $\chi^{2}=314.58, \mathrm{df}=278, \mathrm{p}$ value $=0.06, \chi^{2} / \mathrm{df}=1.13, \mathrm{GFI}=0.94$, AGFI $=$ 0.91 , RMSEA $=0.01$, and measurement model of expressed emotion: $\chi^{2}=78.48, \mathrm{df}=61, \mathrm{p}$-value $=0.06$, $\chi^{2} / \mathrm{df}=1.28, \mathrm{GFI}=0.97, \mathrm{AGFI}=0.93, \mathrm{RMSEA}=0.03$ ). After the overall measurement model was accepted, results of loading t-values and construct validity were examined. In general, based on an accepted level of $0.05, \mathrm{p}<0.01$ and medication use self-efficacy $(\mathrm{r}=0.257, \mathrm{p}<0.01)$. Dysfunctional coping was positively associated with psychotic symptoms $(r=-0.094, p<0.05)$ among schizophrenia patients using methamphetamines. Negatively expressed emotion had positive t-value statistics, which had to be greater than \pm 1.96 before the hypothesis could be rejected. Results indicated that reliability based on confirmatory factor analysis supported the measurement.

\section{Model testing and modification}

After constructing the modification model (Figure 2), the model fit the empirical data and explained $54 \%$ of the variance of psychotic symptoms among schizophrenia patients using methamphetamines $\left(\chi^{2}\right.$ $=8.28, \mathrm{df}=8, \mathrm{p}$-value $=0.41(\mathrm{p}<0.05), \chi^{2} / \mathrm{df}=1.0(<2.00), \mathrm{GFI}=0.99(\geq 0.95)$, AGFI $=0.96(\geq 0.95)$, $\mathrm{CFI}=1.00(\geq 0.95)$, RMSEA $=0.01(<0.05))$. 
http://wjst.wu.ac.th

Table 1 Bivariate relationship among psychotic symptoms, coping, medication use self-efficacy, social support, expressed emotion, stressful life events, and social dysfunction.

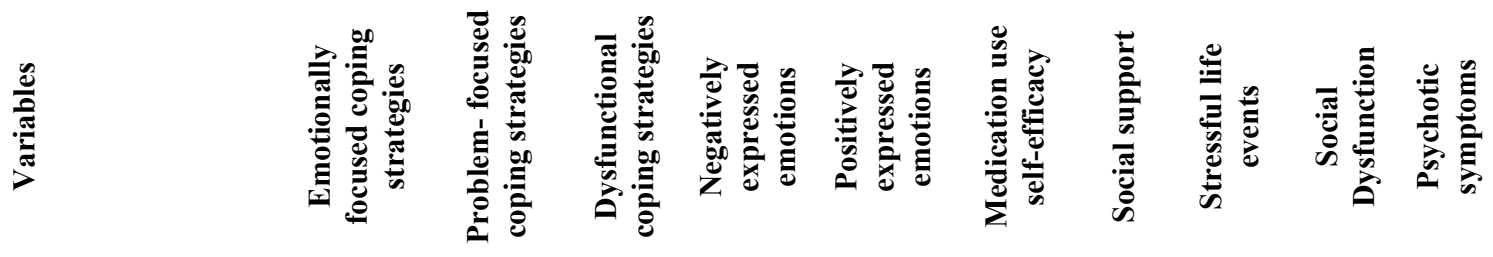

\begin{tabular}{|c|c|c|c|c|c|c|c|c|c|c|}
\hline \multicolumn{11}{|l|}{ Coping } \\
\hline Emotionally focused coping strategies & 1 & & & & & & & & & \\
\hline Problem-focused coping strategies & $.797^{* *}$ & 1 & & & & & & & & \\
\hline Dysfunctional coping strategies & $.568^{* *}$ & $.590^{* *}$ & 1 & & & & & & & \\
\hline \multicolumn{11}{|l|}{ Expressed emotion } \\
\hline Negatively expressed emotions & $.184^{* *}$ & $.286^{* *}$ & $.436^{* *}$ & 1 & & & & & & \\
\hline Positively expressed emotion & .087 & $.151^{* *}$ & $.346^{* *}$ & $.857^{* *}$ & 1 & & & & & \\
\hline Medication use self-efficacy & $.237^{* *}$ & $.257^{* *}$ & $.168^{* *}$ & $.210^{* *}$ & $.175^{* *}$ & 1 & & & & \\
\hline Social support & $.237^{* *}$ & $.194^{* *}$ & -.022 & $-.177^{* *}$ & $-.237^{* *}$ & $.109^{*}$ & 1 & & & \\
\hline Stressful life events & $.291^{* *}$ & $.309^{* *}$ & $.303^{* *}$ & $.250^{* *}$ & $.115^{*}$ & $-.105^{*}$ & -.091 & 1 & & \\
\hline Social dysfunction & $-.099^{*}$ & -.059 & .073 & $.114^{*}$ & $.110^{*}$ & $-.219^{* *}$ & $-.098^{*}$ & $.255^{* *}$ & 1 & \\
\hline Psychotic symptoms & $-.117^{*}$ & -.017 & $.094^{*}$ & $.118^{*}$ & .039 & $-.150^{* *}$ & -.072 & .084 & $.228^{* *}$ & 1 \\
\hline
\end{tabular}

*Correlation was significant at the 0.01 level (1-tailed),

**Correlation was significant at the 0.05 level (1-tailed).

Table 2 Path model of psychotic symptoms with total effect (TE), direct effect (DE), and indirect effect (IE).

\begin{tabular}{|c|c|c|c|c|c|c|c|c|c|}
\hline \multirow{3}{*}{ Exogenous variables } & \multicolumn{9}{|c|}{ Endogenous variables } \\
\hline & \multicolumn{3}{|c|}{ Social support } & \multicolumn{3}{|c|}{ Medication use self-efficacy } & \multicolumn{3}{|c|}{ Psychotic symptoms } \\
\hline & DI & $\mathbf{I E}$ & TE & DI & IE & TE & DI & IE & TE \\
\hline Emotionally focused & & & & & & & $-0.12 * *$ & - & $-0.12 * *$ \\
\hline \multirow[t]{2}{*}{ coping strategies } & & & & & & & $(0.04)$ & - & $(0.04)$ \\
\hline & & & & & & & -0.26 & - & -0.26 \\
\hline Problem- & $0.51 * *$ & - & $0.51 * *$ & $0.21 * *$ & 0.01 & $0.22 * *$ & 0.06 & $-0.02 * *$ & 0.04 \\
\hline focused & $(0.09)$ & - & $(0.09)$ & $(0.04)$ & $0.02)$ & $(0.04)$ & $(0.04)$ & $(0.01)$ & $(0.04)$ \\
\hline coping strategies & 0.41 & - & 0.41 & 0.29 & 0.01 & 0.30 & 0.13 & -0.05 & 0.09 \\
\hline Dysfunctional & & & & & & & $0.07 *$ & - & $0.07 *$ \\
\hline \multirow[t]{2}{*}{ coping strategies } & & & & & & & $(0.03)$ & - & $(0.03)$ \\
\hline & & & & & & & 0.14 & - & 0.14 \\
\hline Negatively expressed & $-0.88 * *$ & - & $-0.88 * *$ & - & -0.01 & -0.01 & 0.02 & 0.00 & 0.02 \\
\hline \multirow[t]{2}{*}{ emotions } & $(0.17)$ & - & $(0.17)$ & - & $0.03)$ & $(0.03)$ & $(0.02)$ & $(0.00)$ & $(0.02)$ \\
\hline & -0.74 & - & -0.74 & - & -0.02 & -0.02 & 0.05 & 0.00 & 0.05 \\
\hline Positively & & & & & & & $-0.04 *$ & - & $-0.04 *$ \\
\hline expressed & & & & & & & $(0.02)$ & - & $(0.02)$ \\
\hline
\end{tabular}




\begin{tabular}{|c|c|c|c|c|c|c|c|}
\hline \multirow{3}{*}{$\begin{array}{l}\text { Exogenous variables } \\
\text { emotions }\end{array}$} & \multicolumn{7}{|c|}{ Endogenous variables } \\
\hline & Social support & \multicolumn{3}{|c|}{ Medication use self-efficacy } & \multicolumn{3}{|c|}{ Psychotic symptoms } \\
\hline & & & & & -0.12 & - & -0.12 \\
\hline Stressful life & & $-0.06 * *$ & - & $-0.06^{* *}$ & 0.01 & $0.01 *$ & 0.01 \\
\hline \multirow[t]{2}{*}{ events } & & $(0.03)$ & - & $(0.03)$ & $(0.02)$ & $0.00)$ & $(0.02)$ \\
\hline & & -0.15 & - & -0.15 & 0.03 & 0.02 & 0.03 \\
\hline Social & & $-0.13 * *$ & - & $-0.13 * *$ & $0.07 * *$ & $0.01 *$ & $0.08 * *$ \\
\hline \multirow[t]{2}{*}{ dysfunction } & & $(0.04)$ & - & $(0.04)$ & $(0.03)$ & $0.01)$ & $(0.03)$ \\
\hline & & -0.17 & - & -0.17 & 0.15 & 0.02 & 0.17 \\
\hline Social & & 0.02 & - & 0.02 & - & 0.00 & 0.00 \\
\hline \multirow[t]{2}{*}{ support } & & $(0.03)$ & - & $(0.03)$ & - & $0.00)$ & $(0.00)$ \\
\hline & & 0.03 & - & $\mathbf{0 . 0 3}$ & - & 0.00 & 0.00 \\
\hline Medication & & & & & $-0.09 * *$ & - & $-0.09 * *$ \\
\hline use & & & & & $(0.03)$ & - & $(0.03)$ \\
\hline self-efficacy & & & & & -0.15 & - & -0.15 \\
\hline $\mathrm{R}^{2}$ & 0.54 & 0.13 & & & 0.12 & & \\
\hline
\end{tabular}

Note $^{*} \mathrm{p}<0.05, * * \mathrm{p}<0.01$

$\left.\left.\left.\left(\chi^{2}: \mathrm{p}<0.05\right), \mathrm{GFI}: \geq 0.95\right), \mathrm{AGFI}: \geq 0.95\right), \mathrm{CFI}: \geq 0.95\right)$, RMSEA $\left.\left.:<0.05\right)\right)[11]$

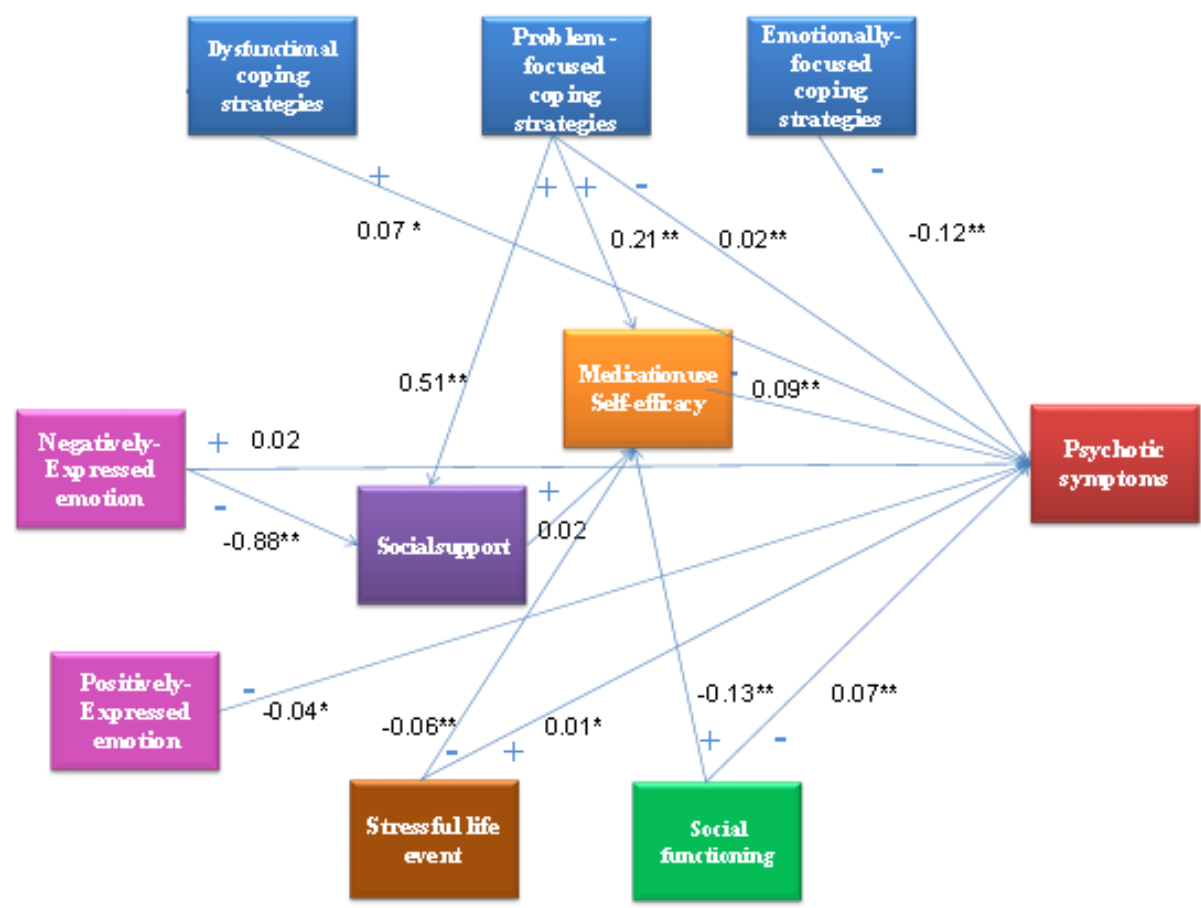

Figure 2 Final model of path analysis of psychotic symptoms among schizophrenia patients using methamphetamines. 


\section{Discussion}

The findings of the path analysis showed that all hypotheses were supported by the empirical data obtained. Interestingly, emotionally focused coping strategies had a direct inverse effect on psychotic symptoms. This confirmed a previous report that the coping strategies most used by participants were emotionally focused coping strategies, such as accepting the reality of what has happened, learning to live with it, getting emotional support from others, getting comfort and understanding from someone, making fun of the situation, trying to see it in a different light to make it seem more positive, and attempting to find comfort in religion or spiritual beliefs such as by praying or meditating. These strategies helped patients adapt to perceived stressors $[19,20]$ and find relief from psychotic symptoms.

Additionally, schizophrenic patients who used methamphetamines appraised information from health care providers and generated understanding about the trajectory of illness and skills in dealing with stress as coping strategies. They used emotionally focused coping strategies to address psychotic symptoms as calls for help and psychological support from health care providers. They perceived providers as people helping them to take medicine daily and who offered love and support through kindness and caring. The findings resembled another study whose participants, when receiving an intervention targeting coping, decreased positive symptom severity [19]. Due to old age and greater experience, this might influence coping strategies used to manage symptoms of schizophrenia. They frequently utilized more proactive coping strategies, such as accessing social support [21].

The study revealed that problem-focused coping strategies have a direct inverse effect on psychotic symptoms through social support and medication use self-efficacy. Participants reported using problemfocused coping strategies to try to improve the situation most often $(40.3 \%)$, followed by emotionally focused coping strategies to accept the reality as it happened $(40.3 \%)$. Positive coping strategies predicted relative decreases in symptoms over time in schizophrenia patients [23, 24]. This finding was different from studies indicating that schizophrenia patients have difficulty dealing with stressful life events $[24,25]$. They may possess a relatively limited repertoire of coping strategies and tend to avoid, rather than actively attempt to solve, problems [26-28].

In addition, medication use self-efficacy had a negative indirect effect $(-0.09, \mathrm{p}<0.01)$ on psychotic symptoms. Strong self-efficacy in taking antipsychotic drugs may decrease positive and negative psychotic symptoms, especially of social withdrawal in schizophrenia in terms of balance neurotransmitters. Using methamphetamines significantly decreases the binding of dopamine to dopamine transporters in the striatum, a brain area important for memory and movement. Biological stressors make individual non-medication adherents. This behavior is the result of dopaminergic stressors leading to changes in cognitive function, including poor judgment, loss of insight, disorganization, and paranoia. Participants appraised information from the psycho-educational group offered by the health care provider and generated knowledge of coping and medication use self-efficacy in terms of problem-solving coping strategies. As a result, problem-solving coping strategies enhanced skills in seeking social support, such as from the health care provider, family, and friends, to maintain and promote medication use selfefficacy in psychotic symptom management [7,29].

The findings were consistent with the vulnerability-stress model of schizophrenia. In schizophrenia patients using methamphetamines, such use may be identified as a biological stressor- a brain toxinmaking them more likely to drop out of treatment and be non-adherent to medication. This behavior can be seen as a result of changes in brain chemistry (such as a dopaminergic stressor), leading to changes in cognitive function, including poor judgment, loss of insight, disorganization, and paranoia.

Participants gained information about the psycho-education group from the health care provider and generated knowledge of coping and medication use self-efficacy for problem-solving coping strategies. Problem-solving coping strategies enhanced skills in seeking social support, such as from the health care provider, family, and friends, to maintain and promote medication use self-efficacy to control psychotic symptoms.

The findings extend evidence of relationships that have been identified in clinical features of schizophrenic patients and methamphetamine abuse. Previous research suggests that dysfunctional coping strategies had a direct positive effect on psychotic symptoms. Participants used many dysfunctional 
coping strategies to address symptoms, using methamphetamines and smoking. Behavioral disengagement, denial, self-distraction, self-blame, and venting were adapted to distressing symptoms. Dysfunctional coping strategies showed high rates of substance abuse among schizophrenia patients, resulting in intoxication; enhancing socialization skills; self-medicating for positive and negative symptoms of schizophrenia; decreasing dysphoria associated with psychotic symptoms; decreasing negative side-effects of antipsychotic medications; and relieving depressive symptoms [30,31].

By contrast, other studies found that individuals did not report abusing substances to counter negative side-effects of psychotropic medications, nor did they report using substances to alleviate any type of psychotic symptom. Schizophrenia patients tended to abuse hallucinogens rather than dopaminergic substances [32].

We found that negatively expressed emotions had a direct positive effect on psychotic symptoms. Empirical data showed that EE was one of the major psychosocial stressors directly associated with the recurrence of illness. The role of $\mathrm{EE}$ on research has consistently established that persons with schizophrenia living with close relatives with negative attitudes are significantly more likely to relapse.

After modifying the model, it was found that negatively expressed emotions had a significant direct effect on social support, perhaps due to the Thai culture of kindness and concern for family members. They perceived that persons with schizophrenia were socially stigmatized, faced with symptom fluctuations sometimes out of their control, needing care from relatives. They always received care even if they were using methamphetamines. Even when caregivers had a negatively expressed emotion toward a patient [33], they still assumed the role of supporter.

In the characteristics of the participants, there was relatively little variation in educational levels. Approximately $61.7 \%$ of participants had a higher educational level than primary school. They had the cognitive function level to know how to ask for social support. Psychotic symptom exacerbations caused stress and protective factors decreased symptom severity through social support. This social support directly helped patients to stabilize emotions about problematic events and recognize self-worth, leading to improved health conditions and behavior. Social support also had a direct effect on the functioning of the immune and endocrine systems, acting as a barrier or buffer, reducing the impact of stress as it generated the feeling of being helped by others.

Positively expressed emotions had a significant direct negative effect $(-0.04, \mathrm{p}<0.05)$ on psychotic symptoms. Expressed emotion was one of the main contributors and robust reliable predictors of psychotic relapse in schizophrenia [34]. Positively expressed emotions included love from family members, regret about illness, good feelings when the patient was happy, and hope that the patient would get better. Analysis showed that 296 (93.3\%) of participants living with their families sensed love and care from family members, a powerful tool to help reduce patient psychotic symptoms.

After the model was modified, it was observed that stressful life events had an indirect effect $(0.01$, $\mathrm{p}<0.05)$ on psychotic symptoms through medication use self-efficacy. Schizophrenia patients experienced psychotic relapses from stressful life events independent of their behavior. Stressful life events may have contributed to a high level of environmental stress interacting with preexisting biological vulnerability factors to boost the likelihood that psychotic symptoms would return [35].

The finding that social function has a positive direct effect on the psychotic symptoms in a population was supported by the current analysis. Repeated hospitalizations often contributed to further social dysfunction [36], indicated by the finding that $83.40 \%$ of participants were readmitted from 2 to 5 times during the period of schizophrenia diagnosis. From another angle, schizophrenia patients using methamphetamines with good social function should manage the time for taking medications, with medication decreasing psychotic symptoms.

\section{Limitations}

Further study might explore other variables such as biological factors, including schizoid personality traits and the level of dopaminergic function. Additional research might examine processes by which families and partners of schizophrenic patients must address limitations associated with this analysis. Generalization of these findings was limited by the participants admitted. Exploring patients 
http://wjst.wu.ac.th

within a community setting would be interesting. Currently, Thai families perceive schizophrenic patients as stigmatized, so local culture may significantly affect social support. In future research, some measure of cognitive function might be obtained as a baseline measure and covariate so that problem-solving factors could be evaluated more accurately.

\section{Conclusions and implication for nursing practice}

The strongest effect on psychotic symptoms is delivered by emotionally focused coping strategies. Medication use self-efficacy, social function, positively expressed emotion, and stressful life events had total and direct effects on psychotic symptoms. The highest indirect effect factors affecting psychotic symptoms were problem-focused coping strategies, stressful life events, and social function. Therefore, psychotic symptoms could be reduced by individual and group nursing interventions that are designed to manage these factors among persons with schizophrenia using methamphetamines. In addition, supportive psychosocial interventions, the combination of pharmacotherapy and psychosocial intervention, has been recommended for treatment of schizophrenia. The best interventions for the acute phase of schizophrenia are psycho-social interventions that reduce psychotic symptoms and in which the individuals can be effectively engaged in treatment. The goals of intervention are to reduce the stress of the patient, provide support for relapse prevention, promote adaptation of the patient to living in the community, and facilitate the continued decrease in symptoms and consolidation of remission.

\section{Acknowledgements}

This study was partially supported by a research grant from Thammasat University, Bangkok, Thailand. Thanks also to all participants for being part of this study.

\section{References}

[1] J Westermeyer. Comorbid schizophrenia and substance abuse: A review of epidemiology and course. Am. J. Addict. 2006; 15, 345-55.

[2] JY Khokhar, LL Dwiel, AM Henricks, WT Doucette and AI Green. The link between schizophrenia and substance use disorder: A unifying hypothesis. Schizophr. Res. 2018; 194, 78-85.

[3] BJ Sadock, VA Sadock and P Ruiz. Kaplan \& Sadock's Synopsis of Psychiatry: Behavioral Sciences/clinical Psychiatry. Wolters Kluwer, Philadelphia, 2015, p. 1273-8.

[4] TL Patterson and OR Leeuwenkamp. Adjunctive psychosocial therapies for the treatment of schizophrenia. Schizophr. Res. 2008; 100, 108-19.

[5] GW Stuart. Principles and Practice of Psychiatric Nursing. Elsevier, Missouri, 2013, p. 433-73.

[6] KH Nuechterlein and ME Dawson. A heuristic vulnerability/stress model of schizophrenic episodes. Schizophr. Bull. 1984; 10, 300-12.

[7] MM Kurtz, RH Olfson and J Rose. Self-efficacy and functional status in schizophrenia: Relationship to insight, cognition and negative symptoms. Schizophr. Res. 2013; 145, 69-74.

[8] CM Hultman, IM Wieselgren and A Öhman. Relationships between social support, social coping and life events in the relapse of schizophrenic patients. Scand. J. Psychol. 1997; 38, 3-13.

[9] NM Docherty, A St-Hilaire, JM Aakre, JP Seghers and AM Cleery and M Divilbiss. Anxiety interacts with expressed emotion criticism in the prediction of psychotic symptom exacerbation. Schizophr. Bull. 2011; 37, 611-8.

[10] N Pujo. 2014, Schizophrenia and Stressful Life Events: Correlations with Negative and Positive Symptoms. Ph. D. Dissertation. The Chicago School of Professional Psychology, California, USA.

[11] BG Tabachnick and LS Fidell. Using Multivariate Statistics. Pearson, Harlow, 2014, p. 666.

[12] P Kittirattanapiboon. Brief Psychiatric Rating Scale (BPRS). Suanprung Psychiatric Hospital, 2001.

[13] CS Carver. You want to measure coping but your protocol's too long: Consider the brief COPE. Int. J. Behav. Med. 1997; 4, 92-100.

[14] P Rapin, A Yupin and T Sureeporn. Medication adherence among persons with post-acute myocardial infarction. Songklanakarin J. Sci. Tech. 2016; 38, 611-20. 
http://wjst.wu.ac.th

[15] W Nardnapa, Y Jintana and S Sunisa. Discriminant Analysis of Relapse and Non-relapse in Thai Muslim Schizophrenic Patients. Master thesis. Chulalongkorn University, Bangkok, Thailand.

[16] H Roohafza, M Ramezani, M Sadeghi, M Shahnam, B Zolfagari and N Sarafzadegan. Development and validation of the stressful life event questionnaire. Int. J. Public Health 2011; 56, 441-8.

[17] S Hanucharurnkul. 1988, Social Support, Self-care, and Quality of Life in Cancer Patients Receiving Radiotherapy in Thailand. Ph.D. Dissertation. Wayne State Uiversity, Michigan, USA.

[18] N Saraswat, K Rao, DK Subbakrishna and BN Gangadhar. The Social Occupational Functioning Scale (SOFS): A brief measure of functional status in persons with schizophrenia. Schizophr. Res. 2006; 81, 301-9.

[19] E López-Navarro, CD Canto, A Mayol, O Fernández-Alonso and E Munar. Psychotic symptoms and quality of life: A mediation analysis of daily-life coping. Psychiat. Res. 2018; 262, 505-9.

[20] C Leclerc, AD Lesage, N Ricard, T Lecomte and M Cyr. Assessment of a new rehabilitative coping skills module for persons with schizophrenia. Am. J. Orthopsychiat. 2000; 70, 380-8.

[21] PT Yanos. Proactive coping among persons diagnosed with severe mental illness: An exploratory study. J. Nerv. Ment. Dis. 2001; 189, 121-3.

[22] PW Corrigan and R Toomey. Interpersonal problem solving and information processing in schizophrenia. Schizophr. Bull. 1995; 21, 395-403.

[23] PW Lee, F Lieh-Mak, KK Yu and JA Spinks. Coping strategies of schizophrenic patients and their relationship to outcome. Br. J. Psychiat. 1993; 163, 177-82.

[24] C Ruzibiza, R E Grattan, R Eder and RJ Linscott. Components of schizophrenia liability are not uniformly associated with stress sensitivity, resilience, and coping. Psychiat. Res. 2018; 260, 10-6.

[25] KT Mueser, DP Valentiner and J Agresta. Coping with negative symptoms of schizophrenia: Patient and family perspectives. Schizophr. Bull. 1997; 23, 329-39.

[26] MK Sharma and P Mahindru. Online methods of managing auditory hallucinations: A new trend to understand psychopathology. Indian J. Psychol. Med. 2017; 39, 354-6.

[27] PH Lysaker, GJ Bryson, RS Lancaster, JD Evans and MD Bell. Insight in schizophrenia: associations with executive function and coping style. Schizophr. Res. 2003; 59, 41-7.

[28] KE Wilder-Willis, PK Shear, JJ Steffen and J Borkin. The relationship between cognitive dysfunction and coping abilities in schizophrenia. Schizophr. Res. 2002; 55, 259-67.

[29] G Singh, P Sharan and P Kulhara. Role of coping strategies and attitudes in mediating distress due to hallucinations in schizophrenia. Psychiat. Clin. Neurosci. 2003; 57, 517.

[30] CJ Asher and L Gask. Reasons for illicit drug use in people with schizophrenia: Qualitative study. BMC Psychiat. 2010; 10, 94-108.

[31] L Wang, SK Lin, YC Chen, MC Huang, TT Chen, SC Ree and CK Chen. Differences in clinical features of methamphetamine users with persistent psychosis and patients with schizophrenia. Psychopathology 2016; 49, 108-15.

[32] M Lammertink, F Löhrer, R Kaiser, M Hambrecht and R Pukrop. Differences in substance abuse patterns: multiple drug abuse alone versus schizophrenia with multiple drug abuse. Acta Psychiat. Scand. 2001; 104, 361-6.

[33] FW O'Connor. A vulnerability-stress framework for evaluating clinical interventions in schizophrenia. Image J. Nurs. Scholar. 1994; 26, 231-7.

[34] T Domínguez-Martínez, C Medina-Pradas, TR Kwapil and N Barrantes-Vidal. Relatives' expressed emotion, distress and attributions in clinical high-risk and recent onset of psychosis. Psychiat. Res. 2017; 247, 323-9.

[35] E Villard, C Vedie, C Lenoir and M Faure. Schizophrenic relapses: Correlation between life events and rehospitalization. Ann. Méd. Psychol. 2015; 173, 443-8.

[36] JY Guo, S Huhtaniska, J Miettunen, E Jääskeläinen, V Kiviniemi, J Nikkinen, J Moilanen, M Haapea, P Mäki, PB Jones, V J eijola, M Isohanni and GK Murray. Longitudinal regional brain volume loss in schizophrenia: Relationship to antipsychotic medication and change in social function. Schizophr. Res. 2015; 168, 297-304. 